CfPA 96-th-09/Submitted to Ap.J.

\title{
A Weak Gravitational Lensing and X-ray Analysis of Abell 2163
}

\author{
G. Squires ${ }^{1}$, D. M. Neumann ${ }^{2}$, N. Kaiser ${ }^{3,7}$, M. Arnaud ${ }^{6}$, A. Babul ${ }^{4}$, H. Böhringer ${ }^{2}$, G. Fahlman ${ }^{5,7}$, \\ and D. Woods ${ }^{5}$
}

\begin{abstract}
We report on the detection of dark matter in the cluster of galaxies Abell 2163 using the weak gravitational distortion of background galaxies, and an analysis of the X-ray emission from the cluster. We find that while the qualitative distributions of the cluster light and the dark matter are similar — shallow and extended, with significant substructure - the X-ray morphology shows a more regular overall appearance. We interpret the joint lensing and X-ray observations as a signature of a merger event in the cluster. We present new ROSAT/HRI data and reanalyze ROSAT/PSPC data, accounting for the effect of a varying background to determine the best fit parameters in the $\beta$-model formalism. We combine the surface brightness fits with two determinations of the radial temperature profile to determine the total mass. Although there are slight variations in the total mass determinations introduced by the uncertainties in the $\beta$-fit, the main contributor to the error arises from the uncertainties in the temperature determinations. Even though the morphologies of the dark matter/light and X-ray gas are quite different, we find that the total mass determined from the X-ray and weak lensing estimates are consistent with each other within the $2 \sigma$ error bars, with the X-ray inferred mass a factor of $\simeq 2$ larger. However, as the lensing mass estimates are differential (the surface density at any point is determined relative to the mean in a control annulus), the shallow, extended nature of the mass profile biases the lensing inferred mass downwards. We estimate the correction for this effect and find very good agreement between the corrected lensing and X-ray results. We determine the gas mass fraction in this cluster and find $f_{g} \simeq 0.07 h^{-3 / 2}$ at all radii and a constant mass-to-light ratio of $M / L_{V}=(300 \pm 100) h M / L_{\odot V}$.
\end{abstract}

\footnotetext{
${ }^{1}$ Center for Particle Astrophysics, University of California, Berkeley, CA, USA 94720

${ }^{2}$ Max-Planck-Institut für extraterrestrische Physik, D-85740 Garching bei München, Federal Republic of Germany

${ }^{3}$ Canadian Institute for Advanced Research and Canadian Institute for Theoretical Astrophysics, University of Toronto, 60 St. George St., Toronto, Ontario, Canada M5S 1A7

${ }^{4}$ Dept. of Physics, New York University, 4 Washington Place, Rm 525, New York, NY, USA 1003-6621

${ }^{5}$ Dept. of Geophysics and Astronomy, University of British Columbia, 2219 Main Mall, Vancouver, BC, Canada V6T $1 \mathrm{Z} 4$

${ }^{6}$ Service d'Astrophysique, CEA Saclay, 91191 Gif-sur-Yvette, France

${ }^{7}$ Visiting Astronomer, Canada-France Hawai'i Telescope. Operated by the: National Research Council of Canada, le Centre National de la Recherche Scientifique de France and the University of Hawai'i
} 
Subject headings: cosmology: observations - dark matter - gravitational lensing - galaxy clusters - X-rays - large scale structure of universe

\section{Introduction}

Determining the masses of the visible and the dark components of the galaxy clusters and mapping out their relative distributions is the key towards understanding both the dynamical state of the clusters and their evolutionary history. As the largest clearly defined objects in the Universe, galaxy clusters are important cosmological probes. They are used, for example, to measure the matter composition of the Universe - e.g., the luminous baryon to dark matter ratio (White et al. 1993) or the mass-to-light ratio (e.g., Blumenthal et al. 1984) on large scales. They are also important tracers of the large scale structure as measured through the mass function or the spatial correlation function (e.g., Efstathiou 1995).

Ideally, one would like to study X-ray bright clusters whose galaxies population has been spectroscopically studied and that also induce measurable, even if only weak, gravitational distortions in the images of faint background galaxies. The X-ray emission of the hot intracluster plasma provides an attractive method to determine cluster masses (Sarazin 1986; Hughes 1989). The derivation of the gravitational mass using X-ray data rests on the assumption, however, that the gas is in hydrostatic equilibrium in the cluster potential. Since the dynamical time scale for the formation of clusters is comparable to the Hubble time and, as many clusters are found to have substructure implying that the are dynamically young and unrelaxed, the X-ray mass derivations are not without uncertainties.

The discovery of gravitational lensing effects, both strong and weak, in galaxy clusters opened a new way to probe the gravitational potential of clusters, which is free of any assumption of the cluster dynamical state (e.g., Fort \& Mellier 1994). Strong lensing effects resulting in obviously distorted images of background galaxies - observed as arcs - were first used to obtain cluster masses by modeling the lens effects. However, such analyses are highly model dependent and are limited to the central regions of the cluster.

The analyses of the weak gravitationally induced distortions in the images of faint, background galaxies, on the other hand, offer a unique opportunity to directly probe the total mass distribution (Tyson et al. 1990; Kaiser \& Squires 1993; see also Squires et al. 1996 and references therein). The weak lensing effects can be measured and inverted to derive the mass distribution in the cluster in a model independent way and free of any assumption of the cluster symmetry and dynamical state. This permits both a non-parametric determination of the total mass, and a 2D map of the total mass distribution in the cluster.

Taken together, the weak lensing analysis and an analysis of X-ray observations offers a unique possibility to probe the relative distributions of the gas and the dark matter and study the dynamical 
relationship between the two. It is worth noting that, on a case-by-case basis, discrepancies in the masses determined by the two methods can naturally arise (for example, if the cluster is strongly elongated cluster along the line of sight). Thus, to be able to make general conclusions about the cluster population, the analyses need to be compared for several clusters for which both good optical and X-ray data are available.

Miralda-Escudé \& Babul (1995) (hereafter MB) performed the first joint X-ray and lensing study of the clusters A2218, A1689 and A2163. In the former two clusters they found that the mass in the central region implied by the observed giant arcs is a factor of 2-2.5 larger than the mass derived from $\mathrm{X}$-ray data if the intracluster plasma is assumed to be isothermal at the observed temperature and in hydrostatic equilibrium in the cluster potential. A similar discrepancy, but not quite as strong, was confirmed by Kneib et al. (1995) also for A2218 in a more detailed effort to model of the strong lensing effect. In contrast, the mass determined from the strong lensing in the regular cluster PKS0745-191 is in agreement with the mass derived from X-ray data, using a multiphase cooling flow model. If it is a common occurrence, the X-ray/lensing mass discrepancy could have important implications for quantities, such as the cluster gas fraction $M_{\text {gas }} / M_{\text {tot }}$, derived solely from X-ray data. Typically, the cluster gas fraction is estimated to be $M_{\text {gas }} / M_{\text {tot }} \geq 0.05 h^{-3 / 2}$ (White \& Fabian 1995; David 1995) a result that has been a source of much discussion (White 1992; Babul \& Katz 1993; White et al. 1993). It is worth noting, however, that the lensing mass determinations based on the strong lensing features are, however, restricted to probing the central regions of clusters.

More recently, Squires et al. (1996) used the weak gravitational lensing distortions to map the mass distribution in A2218 out to a radius of $\sim 0.5 h^{-1} \mathrm{Mpc}$, and jointly analyzed the X-ray, optical and weak lensing results. Under the assumption that the cluster mass distribution extends out to $\sim 1 h^{-1} \mathrm{Mpc}$, as indicated both by the lensing mass profile and the X-ray surface brightness profile, the results suggested that the lensing/X-ray mass discrepancy of kind found by MB may extend systematically beyond cluster core (although the combination of the uncertainties in the lensing and X-ray mass estimates, the latter largely due to poorly determined cluster temperature profile, did not preclude agreement between the two estimates). This discrepancy was recently confirmed by Loewenstein (1996) for both A2218 and A1689 using new ASCA determinations for the temperature profiles. Conversely, in a similar study of the clusters MS1455 and MS0016, Smail et al. (1995) found that the lensing and X-ray masses are in agreement (although we note that the X-ray analyses in these latter cases were based on very sparse $\mathrm{X}$-ray spectroscopic data and thus the uncertainties on the X-ray mass determinations were larger).

In this paper, we present a study of the galaxy, gas, and gravitational mass distribution in the cluster of galaxies A2163 $(z=0.201)$. This cluster is the hottest cluster and one of the two most massive galaxy clusters known so far (Arnaud et al. 1992). Based on GINGA satellite measurements, A2163 has an X-ray temperature of $\sim 14 \mathrm{keV}$ and a X-ray luminosity of $6 \times 10^{45} \mathrm{erg} \mathrm{s}^{-1}$ (Arnaud et al. 1992). It is one of the rare clusters for which the X-ray emission has been traced out to a distance similar to the virial radius; the emission was detected significantly with the ROSAT/PSPC up to $2.3 h^{-1} \mathrm{Mpc}$ or 15 core radii (Elbaz, Arnaud \& Böhringer 1995 - hereafter EAB). While the total mass within that radius, derived from the GINGA and PSPC data, is exceptionally high (2.6 times greater than the total mass of Coma), the corresponding gas mass fraction, $\sim 0.1 h^{-3 / 2}$, is typical of other rich clusters. A quick drop of the temperature at large radii $(\sim 4 \mathrm{keV}$ beyond 6 core radii) was observed recently with ASCA, strongly constraining the total mass profile, assumed to follow a simple parametric law (Markevitch et al. 
1995 - hereafter referred to as MMIYFT). The temperature distribution in A2163 also shows evidence for complex non-azimuthally symmetric temperature variations in the central regions (Markevitch et al. 1994). A2163 exhibits the Sunyaev-Zel'dovich effect (Wilbanks et al. 1994) and is remarkable in the radio, having the most luminous and extended halo yet detected (Herbig \& Birkinshaw 1995). The spatially resolved measurements of the the Sunyaev-Zel'dovich effect, combined with the PSPC data, also confirm the decrease in the temperature in the outer part of the cluster (Holzapfel et al. 1996).

In spite of its exceptional X-ray and radio properties, the dynamical state of A2163 is puzzling. The high mean X-ray temperature and high X-ray luminosity would suggest a massive cluster with a very deep potential well that ought to contain a plethora of strong lensing features such as arcs and arclets. In fact, only two arcs have been observed and they lie at a relatively small distance from a brightest cluster galaxy. This is very much in contrast to the lensing features detected in other hot clusters (e.g., A2218). The optical properties of A2163 are also quite unassuming in comparison to compact clusters such as A2218 and A1689. It is classified as an Abell richness class 2 cluster, its central galaxy is not a cD galaxy, and the cluster galaxy distribution is irregular and extended. On the other hand, A2163 has a very high velocity dispersion $(\sigma=1680 \mathrm{~km} / \mathrm{s})$ and a flat, very extended velocity histogram (Soucail et al. 1996; Arnaud et al. 1994). The optical data, together with the detailed X-ray morphology, have been interpreted as signature of a recent or ongoing merger of two large clusters (EAB; Soucail et al. 1996).

For the present study, we acquired optical images in V- and I-bands using the Canada-France-Hawaii telescope. The total mass distribution of A2163 is determined from the distorted images of the faint background galaxies using the algorithm of Kaiser \& Squires (1993) and amendments (Squires \& Kaiser 1995). We also derive the cluster galaxy light and surface number density distribution. We reanalyze the ROSAT/PSPC data of EAB, allowing for statistical uncertainties in the background, and utilize ASCA temperature information provided by MMIYFT. Furthermore we present new ROSAT/HRI data, which resolves the center of the cluster to a much higher accuracy than the PSPC data. We reinvestigate the total mass and gas mass fraction determination based on X-ray data using the method developed by Neumann and Böhringer (1995) which is free of any assumption on the gravitational potential shape, in contrast with parametric methods used in previous studies of this cluster. We make qualitative comparisons of the morphology of the gas, galaxy and mass distribution and give new insight into the dynamical state of the cluster. We compare the X-ray and lensing total mass estimates, determine the baryon fraction as well as the mass-to-light ratio profile. Finally we consider the cosmological implications of our results.

\section{Optical Data Acquisition and Analysis}

A2163 was observed using the 3.6m Canada-France-Hawai'i telescope on the nights of 1994 June 6-9. The detector used was the $2048 \times 2048$ Loral 3 CCD at prime focus with a pixel size of 0 ' 207 . The observations of A2163 comprised of $3 \times 20$ minute exposures in V and I, with seeing of 0.80 and 0 !'85 (FWHM) in $\mathrm{V}$ and I respectively. We observed the central $7^{\prime}$ of the cluster covering a square of side $\simeq 1 h^{-1} \mathrm{Mpc}$ at the cluster redshift. 
A detailed description of the data reduction procedure for this data sample is given in Squires et al. (1996). Briefly, each bias subtracted image was divided by a median twilight flat. The residual rms scatter in the sky background on the individual images was 24.1 magnitudes per square arcsecond in I, and 25.2 magnitudes per square arcsecond in $\mathrm{V}$. The data was calibrated against photometric standards in the globular clusters M92 and NGC 4147 (unpublished photometry from Davis 1990; see also Stetson \& Harris 1988 and Odewahn et al. 1992) and Landolt (1992) standards in SA110. Color terms were found to be unnecessary in the transformation and the I and V zero points were determined with a formal error of less than 0.005 mag. Using the summed V- and I-band images, we detected 1757 and 2148 objects in $\mathrm{V}$ and I respectively, with a significance of $\nu \geq 4 \sigma$ over the local sky background. The catalogues are complete to a limiting magnitude of 24.4 in $\mathrm{V}$ and 23.0 in I. We measured the position, shape and brightness parameters using our standard procedure (Kaiser, Squires \& Broadhurst 1995) as described in Squires et al. (1996).

\subsection{Cluster Luminosity and Light Distribution}

To obtain a qualitative description of the cluster light distribution, we calculated the cluster galaxy light and surface number density. We combined the $\mathrm{V}$ and I observations to determine the galaxies' color and identified a sequence of red objects with $V-I \simeq 1.5$ (color corrected for reddening by $\mathrm{E}(\mathrm{V}-\mathrm{I})=0.2$; see Bessell \& Brett 1988), which we classified as candidate cluster galaxies. To extract this color-selected subsample, we fitted a linear model to the color sequence and identified bright objects $(I<21)$ with color within 0.2 magnitudes of the mean.

We display the color-selected cluster galaxy light distribution and surface number density as contour plots placed on the rebinned V-band image of the cluster in Figure 1. The contours have been smoothed with a Gaussian filter with scale 0!66. The galaxy light distribution shows three peaks: one centered on a giant elliptical galaxy near the center of the frame - this is the galaxy that has two associated arcs $15{ }^{\prime \prime} 6$ to the west. Two other peaks in the galaxy light distribution appear to the northeast and southwest of this galaxy, and there is an extension towards the western edge of the image. The galaxy number density distribution is broadly similar, with the main peak slightly displaced from the central giant elliptical galaxy and a second peak about 2.5 to the west. Together, these plots give the impression of a somewhat irregular and extended cluster - the relative strengths of each light concentrations are comparable. Qualitatively, this is also apparent from a visual inspection of the image: there are several prominent luminous galaxies distributed over the frame, with no obvious dominant galaxy defining an optical center. Spectroscopy of this field confirms the very flat and extended nature of the galaxy distribution in this cluster Arnaud et al. 1994 Soucail et al. 1996).

We formed three estimates of the cumulative light profile in the cluster, taking the giant elliptical galaxy as the cluster center. The light profiles are displayed in Figure 2. The short-dashed line shows the result of considering only the light associated with the color-selected galaxy sequence. The solid-line shows the profile obtained by adding the light, as determined from aperture magnitudes, associated with all the galaxies detected in the image. To account for the possibility that there is extended intracluster light present, or light in extended galaxy halos, we also calculated the total light 

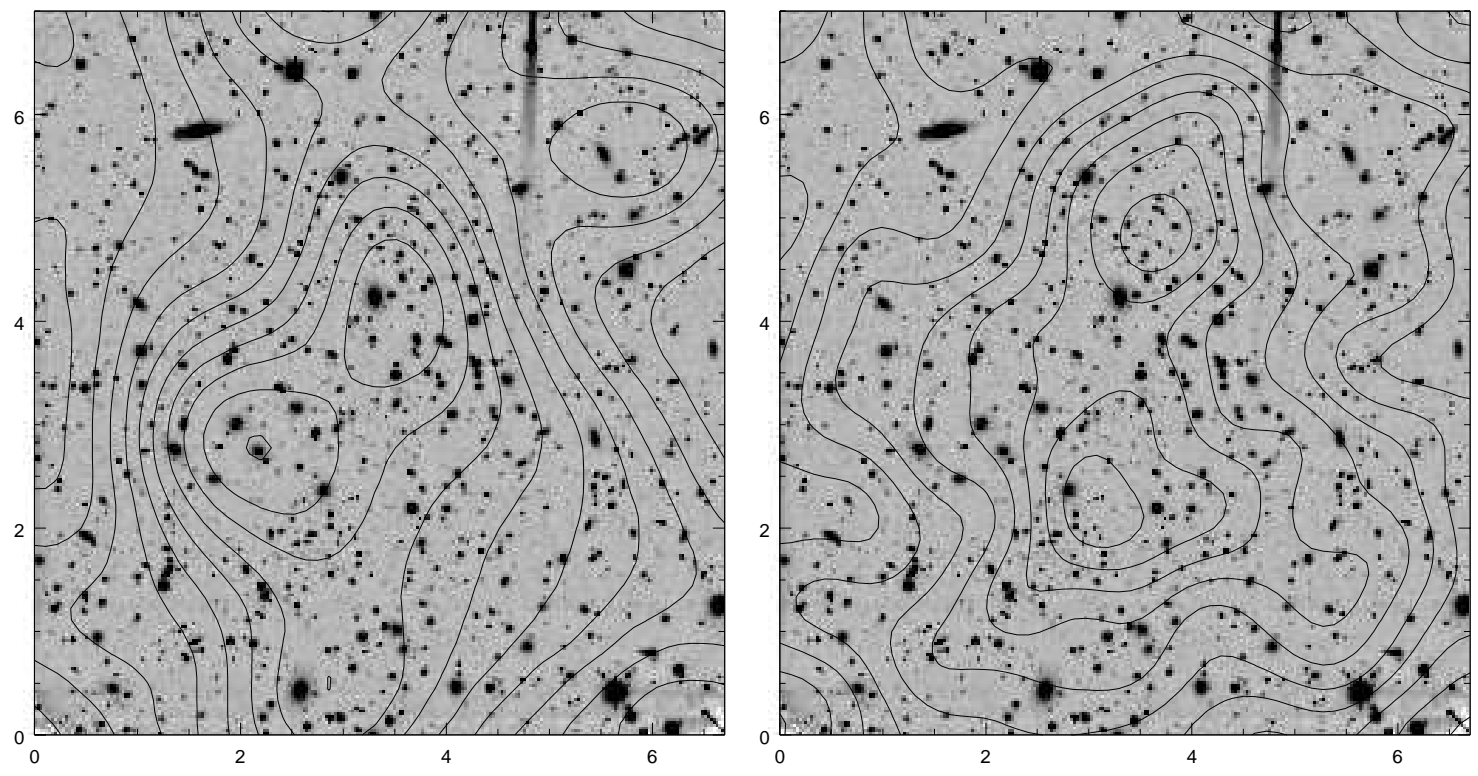

Fig. 1. - The luminosity and number weighted contours of the color-selected cluster galaxy sample placed on the rebinned V-band image of the cluster. The left plot shows the luminosity distribution and the right panel is the galaxy number distribution. The contours have been smoothed with a Gaussian filter with scale 0!66. North is to the right: East is up. The angular scale is arcminutes $\left(1^{\prime}=0.127 h^{-1} \mathrm{Mpc}\right)$. 


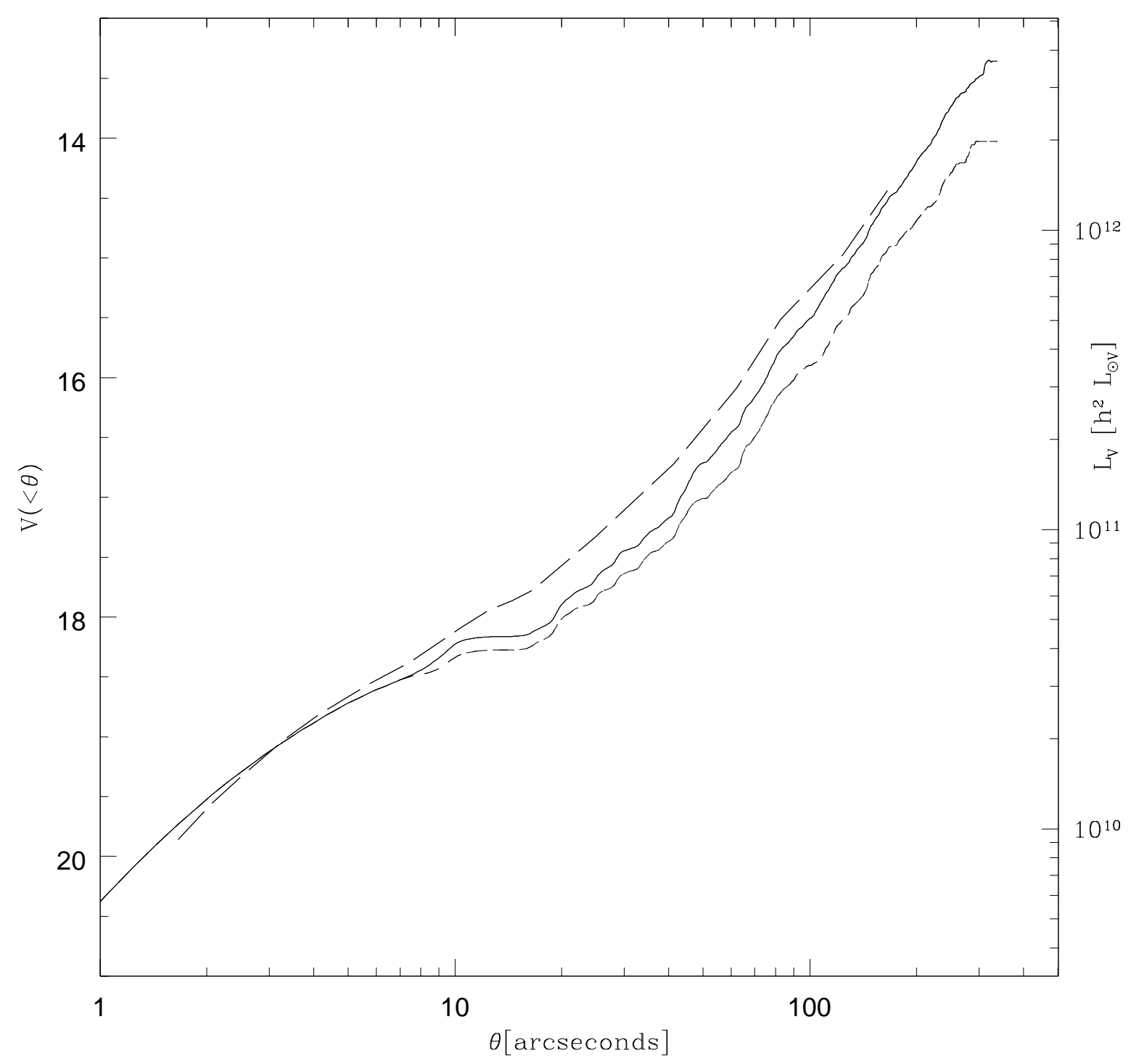

Fig. 2.- The cumulated V-magnitude and $L_{V}$ luminosity for A2163. The solid lines come from adding the light from all galaxy candidate objects in the field. The short-dashed line is for the color-selected sequence and the long-dashed line is the 'total' light in the cluster calculated by adding all the light on the image after masking cosmic rays and stars. The scale is $60^{\prime \prime}=0.127 h^{-1} \mathrm{Mpc}$. 
directly from the images, masking only cosmic rays and stars and adding all the light from the cleaned image. The apparent magnitudes associated with the galaxies were converted into luminosity using the transformation $L_{V}=10^{0.4\left(M_{V \odot}-V+D M+K+A_{v}\right)} L_{\odot}$, where $M_{V \odot}=4.83$ is the total solar $\mathrm{V}$ magnitude and $D M=39.00$ is the distance modulus for A2163 in $\Omega=1, h=1$ cosmology (in computing the light profiles, we assumed that all sources were at the cluster redshift). We also applied a K-correction of $K=0.4$ as suggested by the data in Coleman, Wu and Weedman (1980) and used an extinction correction of $A_{v}=0.54$.

The resulting profile is shown as the long-dashed line. In spite of the fact that the total light profile exceeds that associated with the cluster sequence subsample by $\simeq 30 \%$ (the total light profile also includes contribution from background and foreground galaxies), all three estimates exhibit similar trend as a function of radius. At angular radius $\theta>1^{\prime}$ from the central elliptical galaxy, the cumulative light profiles rise as $\theta^{1.6}$, implying that the azimuthally-averaged surface brightness profile of the cluster is $l(\theta) \propto \theta^{-0.4}$. This surface brightness profile is shallower than isothermal $\left(\propto \theta^{-1}\right)$.

\subsection{Lensing Analysis}

Since the seeing conditions for both the I- and V-band observations were similar, we employed both data sets for the lensing analysis. The I-band images do have somewhat higher sky noise than the V-band images and hence, the corresponding results are slightly noisier.

We selected as candidate background galaxies all objects with half-light radius greater than 1.2 times the mean stellar half-light radius and applied a bright magnitude cut $(V<22$ and $I<20.5)$ to reduce foreground and cluster galaxy contamination. This cut is somewhat arbitrary - the values quoted above were chosen after some experimentation to maximize the signal-to-noise in the reconstructions (although the change is rather small if the above cuts are increased by $\simeq 1$ magnitude). With these selection parameters, we found 736 galaxies in the I-band data and $690 \mathrm{in} \mathrm{V}$.

Before converting the measured galaxy shapes into estimates of the cluster surface density, we corrected for two effects: The anisotropy in the point spread function (psf) due to wind-shake, guiding errors, etc., and the smoothing due to seeing. The procedure to correct for these systematic errors is described fully in our analysis of A2218, (Squires et al. 1996). Briefly, we mapped the stellar psf across each image and applied an empirical correction (Kaiser, Squires \& Broadhurst 1995) to each object to remove the anisotropy in the psf. We found a residual stellar ellipticity of less than $0.5 \%$ after the correction had been applied. Similarly, the effect of seeing was calibrated by simulations using the HST-MDS data. We applied a constant shear to the images and created a simulated data set that had the same pixel resolution, exposure time, sky noise, and seeing conditions as the data observed at CFHT. We then processed the simulated data in an identical fashion to the CFHT data to determine the signal loss due to seeing. We repeated this simulation a number of times, applying different shear and random sky noise realizations and averaged the signal recovery fraction.

The seeing and psf-corrected galaxy shape measurements were converted into the cluster's surface 
mass density distribution using the maximum probability extension (Squires \& Kaiser 1995) to the original Kaiser \& Squires (1993) algorithm. The calculation used 15 wavemodes and a regularization parameter of $\alpha=0.05$ (changing this value by factors of 0.5 to $2 \times$ changes the amplitude of the results by $\simeq 4 \%$ ). In Figure 3 we display the mass surface density reconstruction for Abell 2163. The results for both the V- and I-band data are shown.

The contour maps of the mass distribution in Abell 2163 is rather irregular and quite different from the more symmetric distributions that we have seen in other clusters (eg. A2218 - see Squires et al. 1996). The V-band mass map shows two mass peaks, with possibly a third one in the northeast corner of the image. One of the mass peaks appears to be associated with the giant elliptical galaxy that has two arcs nearby. The significance of each of the peaks, however, is rather modest $(\leq 3 \sigma)$ and is lower than that we have found for other clusters such as A2218. This is not surprising given the small number of galaxies used in the reconstructions $(\mathrm{N} \simeq 700)$ and the moderate seeing conditions. Furthermore, the amplitude of the shear is quite small (see Figure 1 ) which naturally arises if the surface mass distribution is flat. It does, however, mean that the precise location of the peaks should be interpreted with caution. The I-band mass map also features two main mass peaks and like the V-band mass map, shows that the cluster mass distribution is elongated primarily along the East-West direction, with two main mass concentrations. We attribute the small scale differences in the V-and I-band mass maps to a combination of mass peaks of modest significance and the higher sky noise affecting the I-band data. (It is possible that the eastern peak with northern extension that appears in the I-band data is the combination of the eastern and northeastern peaks that appear in the V-band). Taking the average of the two maps yields a result that is smoother, but still shows the bimodal structure, again with only moderate statistical significance (the "averaged" peaks have significance of $3 \sigma$ and $2.5 \sigma$ respectively).

The small scale fluctuations between the V- and I-band results noted above have little impact on the global statistics, such as the gravitational shear profile and the cluster radial mass profile, that we are interested in and which we can determine with higher significance. We display in Figure 1 (top panels) the azimuthally-averaged, mean radial shear profile for A2163 computed about the giant elliptical galaxy with associated arcs. We have also done the calculations with the centroid of the X-ray emission chosen as the cluster center in order to facilitate comparisons with results derived from the X-ray observations. The results within $\theta \simeq 1^{\prime} \simeq 130 h^{-1} \mathrm{kpc}$ should be interpreted with care; any contamination by the cluster (or foreground) galaxies of the catalog of faint galaxies used to determine the shear profile will tend to dilute the signal and lower the measured shear. The problem is of particular concern in the present case due to the small number of faint galaxies available for determining the gravitational shear. Fortunately, the contamination only tends to affect shear measurements for the central regions in the cluster. In our analyses of A2218 (Squires et al. 1996) and MS 1224 (Fahlman et al. 1994), we found that the contamination mostly affected results for the innermost $\sim 100 h^{-1} \mathrm{kpc}$, but is negligible at larger radii.

For comparative purposes, we also show in Figure 1 the expected shear for a softened isothermal-like surface density distribution, $\Sigma(\theta) \propto \sigma^{2}\left(\theta^{2}+\theta_{c}^{2}\right)^{-1 / 2}$, where $\sigma$ is the line of sight velocity dispersion and $\theta_{c}$ is a softening (core) parameter. We consider three cases: In two of the cases, we use the measured velocity dispersion $\sigma=1680 \mathrm{~km} / \mathrm{s}$ and show the results for a singular distribution, $\theta_{c}=0$ (dashed curve), and a distribution where the core radius is set equal to that of the cluster's X-ray surface brightness distribution, $\theta_{c}=1$ '.2 (solid curve). In comparing the observed and the model shear profiles, 


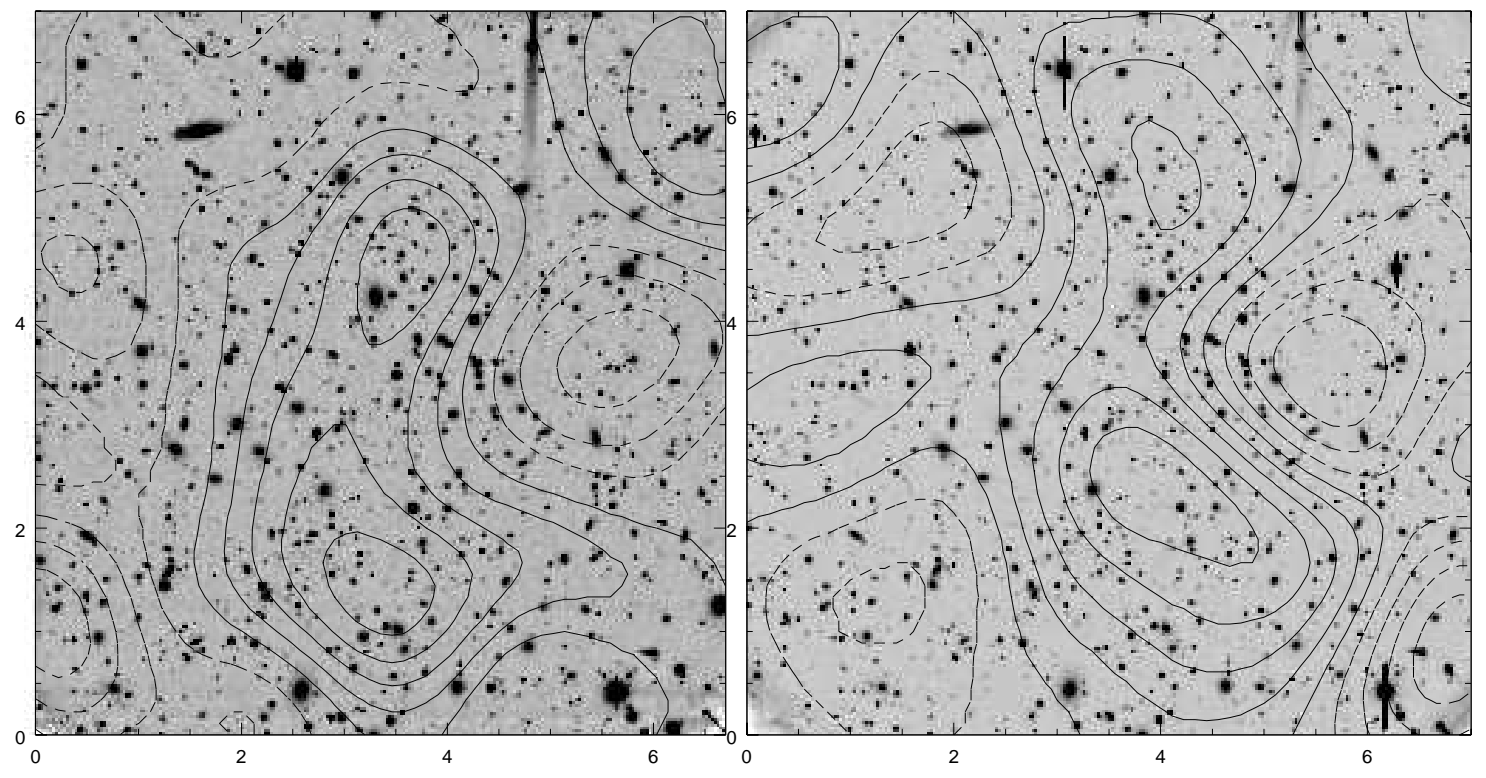

Fig. 3.- The reconstructed surface densities placed on the rebinned V-band image of the cluster. The V- and I-band results are shown in the left/right panels respectively. The contours have been smoothed with a 0'66 Gaussian filter. North is to the right; East is up and the angular scale is arcminutes $\left(1^{\prime}=0.127 h^{-1} \mathrm{Mpc}\right)$. 
it is clear that the observed shear profile is much weaker than that induced by a singular isothermal profile characterized by a velocity dispersion comparable to that observed for the cluster galaxies, even if one takes into consideration the possibility that shear in the inner $1^{\prime}$ might be higher than observed. On the other hand, a mass distribution also characterized by $\sigma=1680 \mathrm{~km} / \mathrm{s}$ but with a core radius comparable to that observed in the X-ray produces less shear than observed, particularly in the inner $1^{\prime}$. In fact, such a profile would not be able to produce the thin large arcs observed in the vicinity of the giant elliptical galaxy. Even if the cluster surface mass is flat over the range $1^{\prime}<\theta<2^{\prime}$, both the observed shear profile and the thinness of arcs suggest that the cluster mass profile must steepen in the central regions.

We also consider a singular model that provides a reasonable fit to the measured shear (dot-dashed curve). This model corresponds to $\sigma=740 \mathrm{~km} / \mathrm{s}$ although a singular isothermal model with $\sigma$ as high as $1000 \mathrm{~km} / \mathrm{s}$ is also consistent with the observations, particularly if one takes into account the possible suppression of shear in the inner regions of the cluster by contamination. Indeed, under the simple assumption of a spherical, isothermal mass distribution, a velocity dispersion of $\simeq 930 \mathrm{~km} / \mathrm{s}$ would be required to form the Einstein radius at the observed arc position.

In the bottom panels of Figure 1 , we display the statistic (Kaiser et al. 1995)

$$
\zeta\left(\theta_{1}, \theta_{2}\right)=2\left(1-\theta_{1}^{2} / \theta_{2}^{2}\right)^{-1} \int_{\theta_{1}}^{\theta_{2}} d \ln (\theta)\left\langle\gamma_{t}\right\rangle,
$$

which measures the azimuthally-averaged, mean surface density interior to $\theta_{1}$ relative to the mean in an annulus $\theta_{1}<\theta<\theta_{2}$. The outer radius of the control annulus was fixed at $\theta_{2}=4^{\prime}$.6. Displayed as well are the curves associated with the three isothermal-like surface mass distributions discussed above.

Based on the observed radial shear profile and corresponding $\zeta$ statistic, it is clear that the azimuthally-averaged radial mass surface density at angular distances $\theta>1^{\prime}$ is not steeper than isothermal $\left(\theta^{-1}\right)$ and in fact, is likely to be shallower, consistent with the flat light surface brightness profile.

To convert the $\zeta$ measurements into mass estimates, we estimate $\Sigma_{c r i t}=\left(4 \pi G D_{l} \beta\right)^{-1}$, where $D_{l}$ is the angular diameter distance to the lens and $\beta=\max \left(0,\left\langle 1-w_{l} / w_{s}\right\rangle\right)$. In an Einstein de-Sitter universe with $\Omega=1$, the comoving distance $w$ is defined as $w=1-1 / \sqrt{1+z}$. We determine $\beta$ by an extrapolation of faint redshift surveys (Lilly 1993; Lilly 1995; Tresse et al. 1993) to fainter magnitudes and find, for A2163 and our background galaxy selection criteria, $\Sigma_{\text {crit }}=(6.7 \pm 0.6) \times 10^{15} \mathrm{~h} \mathrm{M}_{\odot} / \mathrm{Mpc}^{2}$. In Figure 5 we display the radial mass profile for Abell 2163 with the giant elliptical galaxy taken as the cluster center. The results from the V-band data are displayed in the bottom panel and the I-band results are shown in the top panel. We also mark the mass determined by MB assuming the arcs lie at the Einstein radius and plot the curves corresponding to the three isothermal-like mass distributions.

Once again, the comparison of the derived mass profile and those corresponding to the isothermallike models must be undertaken with care. As discussed previously, the dilution of the shear signal in the central regions $\left(\theta<1^{\prime}\right)$ results in the mass estimate in the central regions being biased downward. In addition, the mass estimates derived from the lensing analysis are differential in nature - the surface density at any point is measured relative to the mean in the control annulus. Hence, if the 

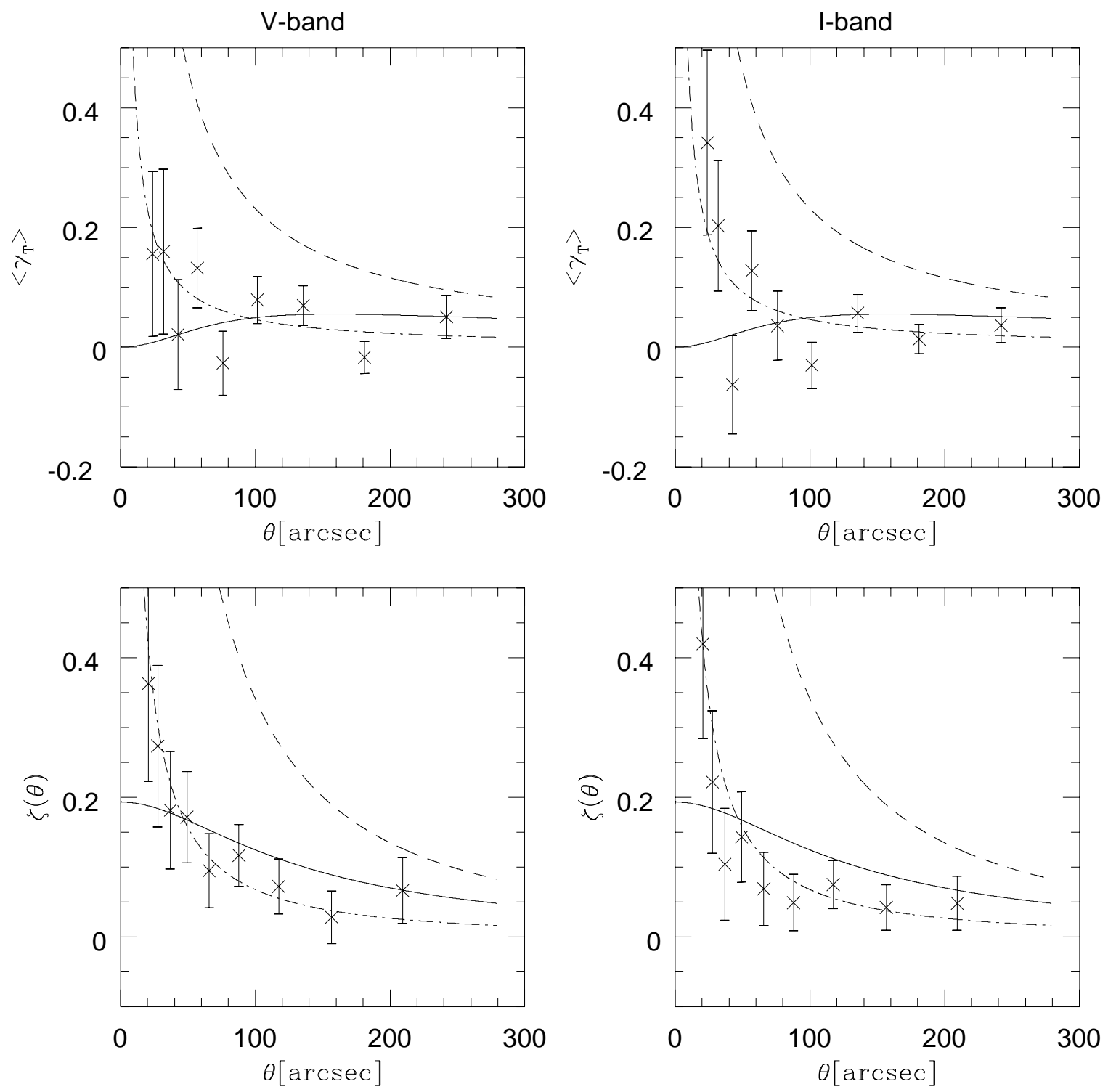

Fig. 4.- The top panels show the azimuthally-averaged, mean radial shear about the giant elliptical galaxy centroid. The bottom panels show the $\zeta$ statistic. The V and I-band results are shown in the left and right columns respectively. The solid line is the prediction for a softened isothermal model with the measured velocity dispersion of $\sigma=1680 \mathrm{~km} / \mathrm{s}$ and a core of 1.2 (which corresponds to the X-ray core radius). The dashed line is the prediction in the singular isothermal model with the same velocity dispersion. The dot-dashed line is the best-fit singular isothermal model $(\sigma=740 \mathrm{~km} / \mathrm{s})$. The scale is $60^{\prime \prime}=0.127 h^{-1} \mathrm{Mpc}$. 

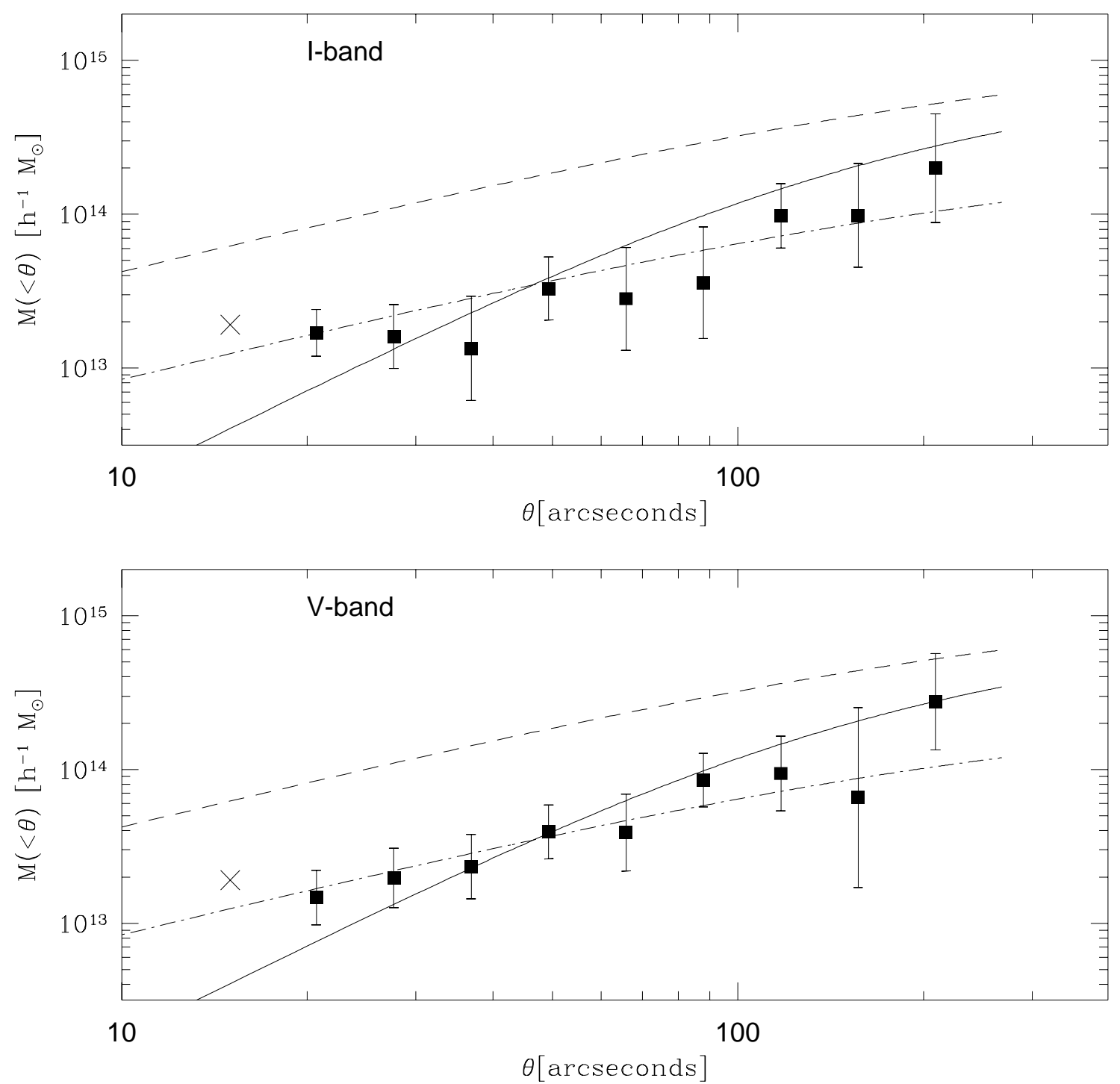

Fig. 5.- The radial mass profiles for A2163 for the V- and I-band data (bottom and top panels respectively) with the center chosen to be the giant elliptical galaxy. The mass is a cumulative function, and hence the uncertainties on different points are correlated. The lines are the model predictions with parameters as described in Figure 4 . In these calculations, we have corrected for the mass in the control annulus to facilitate direct comparison with the lensing masses derived using the $\zeta$ statistic. The " $\mathrm{x}$ " represents the mass determined by assuming the arcs lie at the Einstein radius. The errors on the weak lensing points were determined by adding the errors in $\kappa$ and $\Sigma_{\text {crit }}$ in quadrature. The outer radius of the control annulus was fixed at 4.'6. The scale is $60^{\prime \prime}=0.127 h^{-1} \mathrm{Mpc}$. 
cluster mass distribution extends into the control region - a likely scenario given that the field of view being analyzed is relatively small, the cluster light profile continues to rise and that the X-ray surface brightness profile extends to $\theta \approx 15^{\prime}$ - the lensing mass estimates will underestimate the true mass by some significant amount. We attempt to address this latter issue when we make comparisons with the mass profile derived from the X-ray observations (see \$4.). Note, however, that the mass estimate of MB is not subject to these uncertainties.

Using the cluster light and the lensing mass profiles, we can compute the cluster mass-to-light ratio as a function of radius. To account for the subtraction of the material in the control annulus in the mass estimator, we form an analogous quantity, $\zeta_{L}$, for the light. We calculate the mean light surface density at each radius relative to the mean in the control region. Under the assumption that mass traces the light - the cluster light and the lensing mass profiles are consistent with this assumption, the ratio of the $\zeta$ values based on the observed shear and $\zeta_{L}$ forms an unbiased estimate of the mass-to-light ratio in the cluster. This ratio is plotted in Figure 6. The light estimate comes from using all the light associated with the galaxies detected in the image (which, as we have discussed previously, probably includes a contribution from galaxies not associated with the cluster). We find a mass-to-light ratio of $M / L_{V}=(300 \pm 100) h M_{\odot} / L_{V \odot}$ at $\theta \approx 2^{\prime} \approx 0.250 h^{-1} \mathrm{Mpc}$, with the radial profile being consistent with a constant $M / L_{V}$ across the cluster.

\section{X-ray analysis}

Abell 2163 was observed by ROSAT for 12133s in total with the PSPC (in two observations) and for 36194s with the HRI. Figure 7 shows the HRI image of A2163 superimposed on the rebinned V-band image of the cluster. The higher resolution HRI data confirm the results of the PSPC observation, as discussed in EAB: The centroid of the X-ray emission is not associated with any obvious optical counterpart - the peak of the emission lies about $1^{\prime}$ west of the giant elliptical galaxy with the arcs. The central region shows an elongation in the east-west direction, roughly along the direction defined by the two main concentrations in the optical and total matter distributions. The X-ray morphology, however, appears to be much more regular than the galaxy or total mass morphology, displaying clear concentric isophotes, that are elongated roughly in the same direction as the central part of the cluster. As the HRI provides a much higher spatial resolution it shows the innermost part of the cluster in more detail than the PSPC data. Interestingly, there is an indication of bimodality in the inner arcminute. This substructure, which was not resolved by the PSPC, is aligned with the large scale elongation axis.

To quantify the total mass distribution we proceed in the standard fashion. We fit an isothermal $\beta$-model (Jones \& Forman 1984), convolved with the instrument PSF, to the surface brightness distribution in the ROSAT/PSPC image. This allows an analytical deprojection of the surface brightness profile, $S(r)$, into a gas density distribution $\rho(r)$ :

$$
S(r)=S_{0}\left(1+\frac{r^{2}}{r_{c}^{2}}\right)^{1 / 2-3 \beta}+B
$$




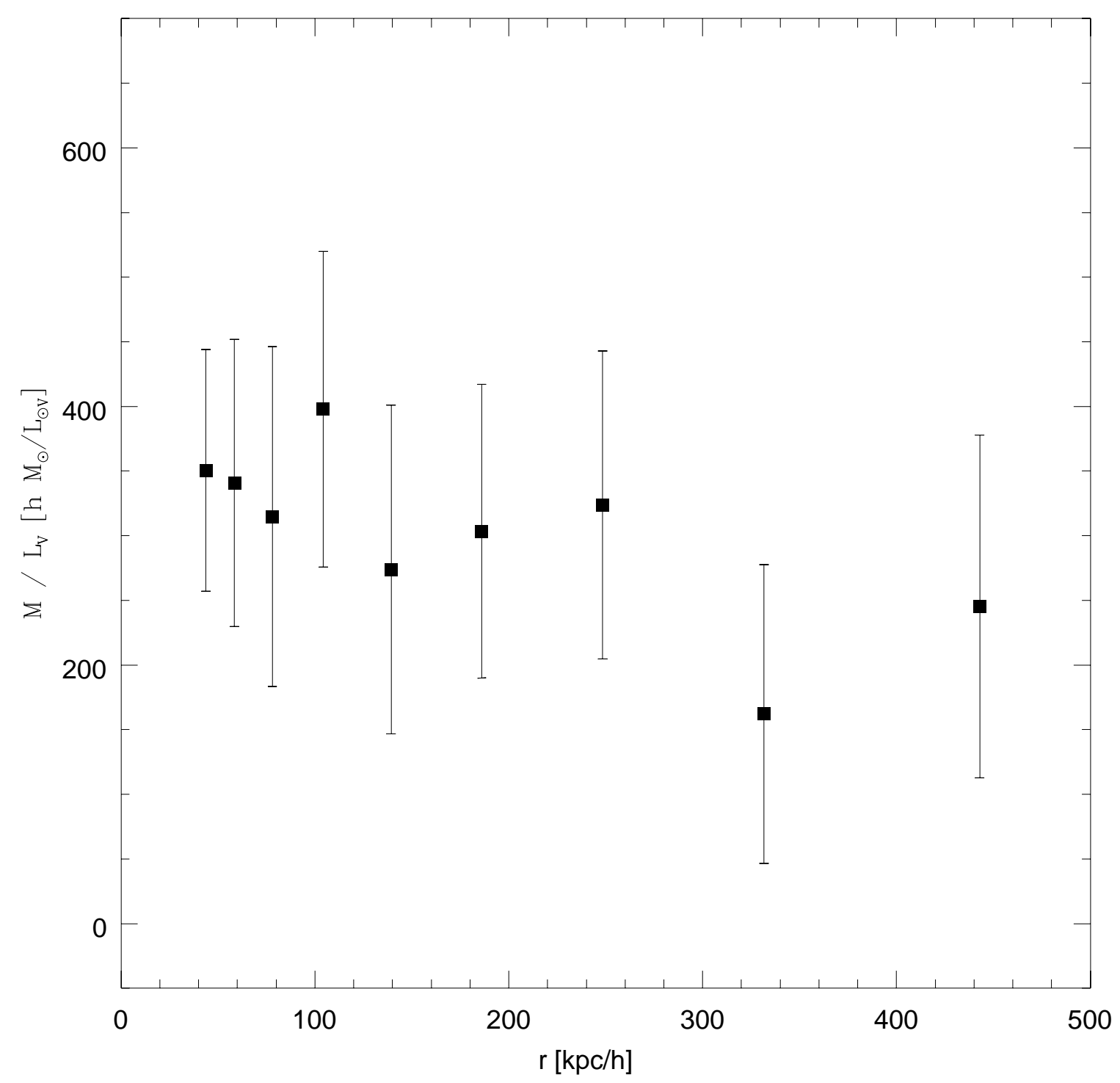

Fig. 6. - The mass-to-light $M / L_{V}$ ratio for A2163 versus radius from the giant elliptical galaxy. The light estimate comes from using all the light associated with galaxies detected in the image (including potential contributions from galaxies not associated with the cluster). The mass-to-light ratio was formed by calculating the mean surface mass and light densities as a function of radius, relative to the mean in the control annulus. Both quantities are cumulative and thus the uncertainties on different points are correlated. 


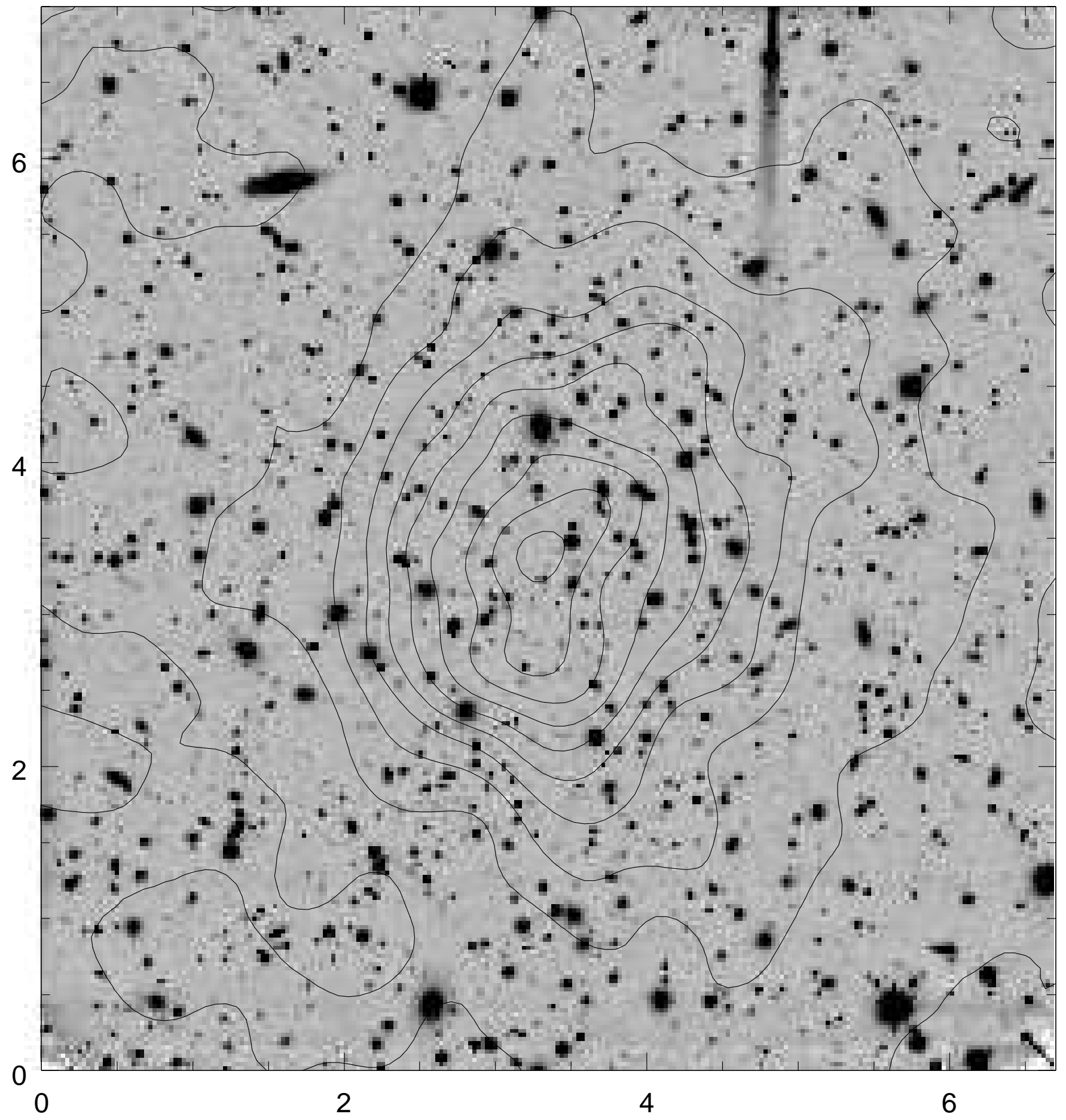

Fig. 7. - The ROSAT HRI image of the X-ray emission (contours) placed on the optical image of A2163. The contours are smoothed with a Gaussian filter with scale $12^{\prime \prime}$. The contour levels are linearly spaced by $7 \times 10^{-8}$ counts $/ \mathrm{s} / \mathrm{sq}$. arcsecond with the maximum contour corresponding to $8.9 \times 10^{-7}$ counts $/ \mathrm{s} / \mathrm{sq}$. arcsecond. North is to the right; East is up. The angular scale is arcminutes (or $127 h^{-1} \mathrm{Mpc}$ ). 


$$
\rho(r)=\rho_{0}\left(1+\frac{r^{2}}{r_{c}^{2}}\right)^{-3 \beta / 2}
$$

Formally, this inversion assumes that the gas is isothermal and spherically symmetric. However, since the ROSAT sensitivity varies only very weakly with the emission temperature (6\% from $2 \mathrm{keV}$ to $10 \mathrm{keV})$ (Jones \& Forman 1992; Böhringer 1994) the surface brightness fit is still a very good representation of the gas density profile even though the cluster is not isothermal.

Assuming a constant background, $B, \mathrm{EAB}$ obtained for the fit-parameters $\beta=0.620_{-0.012}^{+0.015}$ and $r_{c}=(153 \pm 10) h^{-1} \mathrm{kpc} \equiv 1^{\prime} \cdot 20 \pm 0$ 0.075. These parameter error estimates only take into account the statistical uncertainties on $B$. We reexamine these results allowing for systematic errors in the background. Such errors can occur due to either improper vignetting correction, the contribution of the support structure of the X-ray telescope, or due to the contribution from unresolved point sources. The latter maybe caused by the fact that in areas with large off-axis angles, the PSF of X-ray telescopes usually increases by a substantial amount. These background variations of the PSPC data, derived by EAB from a study of the fluctuation in the counts, are of the order of $9 \%$. Including these uncertainties, by fitting the data with a background $\pm 10 \%$ and adding this systematic error in quadrature to the error estimates obtained by $\mathrm{EAB}$, we obtain new errors for $\beta$ and for $r_{c}$, so that $\beta=0.620 \pm 0.035$ and $r_{c}=(153 \pm 16) h^{-1} \mathrm{kpc} \equiv 1 ! 20 \pm 0$ ' 13.

In addition to the PSPC data we analyzed the data of the HRI observation. These provide a higher resolution of the central area of the cluster, which is relevant for comparison with the weak lensing analysis done here. The HRI data also permit a test of how the derived mass profile is affected by the limited resolution of the PSPC (we expect the effect to be small since the PSF, which is included in the above analysis, has a FWHM of $20^{\prime \prime}$ compared to the 1.2 core radius). The downside is that the HRI detector has a higher internal background, so that the cluster emission can only be traced out to approximately $1 h^{-1} \mathrm{Mpc}$, compared to more than $2 h^{-1} \mathrm{Mpc}$ with the PSPC. Fitting the HRI data we obtain $\beta$ and $r_{c}$ values consistent with the PSPC results: $\beta=0.57$, and $r_{c}=142 h^{-1} \mathrm{kpc}$ with errors of $\simeq \pm 10 \%$. Due to the lower sensitivity and the higher background of the HRI, these errors are larger than the errors for the PSPC fit including the background variations.

By combining the fits from the isothermal $\beta$-model of EAB with the temperature profile, and employing the assumptions of spherical symmetry and hydrostatic equilibrium, we determine the total mass via the relation

$$
M(<r)=-\frac{r^{2} k T}{G \mu m_{p}}\left(\frac{d \ln \rho}{d r}+\frac{d \ln T}{d r}\right) .
$$

In addition we estimate the gas mass of the cluster by integrating the density given in equation 3-1, under the assumption of spherical symmetry. We discuss the uncertainties in the mass estimate introduced by the above assumptions in Section 3.1. Here, we simply note that at present, the uncertainties in the mass estimate are dominated by large uncertainties in the temperature. A more detailed justification is given below.

Two different azimuthally averaged, radial temperature profiles of the ICM are considered for the determination of the total mass. We first consider the results obtained by MMIYFT from a combined fit of the ASCA/GIS and SIS data: $11.0 \mathrm{keV}$ to $14.1 \mathrm{keV}$ within the innermost $445 h^{-1} \mathrm{kpc}, 8.6$ to $14.2 \mathrm{keV}$ for $445 h^{-1} \mathrm{kpc}<r<953 h^{-1} \mathrm{kpc}$, and 2.9 to $4.9 \mathrm{keV}$ for $953 h^{-1} \mathrm{kpc}<r<2 h^{-1} \mathrm{Mpc}$ (see also Table 1). 
MMIYFT noticed that the overall GINGA best fit temperature is higher than the one just mentioned. To probe the effect of this, we considered a second temperature profile that comes from the combined ASCA/GINGA fit of MMIYFT. The resulting increased temperature may resemble more the real temperature profile of A2163 as in principle the GINGA LAC instrument is more reliable for the determination of such high temperatures, due to its higher sensitivity especially at high energies. To assess the possible impact of these systematic differences (which are of the order of the statistical errors) on the mass estimate, we used the following temperature profile: a temperature of 10.5-16.2 keV within $445 h^{-1} \mathrm{kpc}$, to $8.6-18.4 \mathrm{keV}$ between 445 and $953 h^{-1} \mathrm{kpc}$, and $2.9-5.5 \mathrm{keV}$ up to a radius to $2 h^{-1} \mathrm{Mpc}$ (see also Table 11). This profile is centered on the best fit values deduced by MMIFYT from their simultaneous fit of SIS, GIS and GINGA data and the errors are conservatively set such as to encompass all the allowed values found by MMIFYT from the different instruments.

To account for these substantial uncertainties of the temperature profile in the mass determination we use the method developed by Neumann \& Böhringer (1995). It is based on a Monte-Carlo technique that translates errors in temperature into errors of the total mass. This is critical as the uncertainties in temperature are the main source of uncertainty in the final results, and the gradient of the temperature enters the mass equation. The method has the further advantage, over parametric methods used in previous studies (EAB; MMIYFT) for this cluster, to be free of any assumption on the radial potential shape. The method calculates possible temperature profiles within the given temperature range determined from the X-ray spectroscopy in different rings and includes the additional constraint that the resulting mass profile has to increase with radius. We use a stepwidth of $160 \mathrm{~h}^{-1} \mathrm{kpc}$, and calculate $10^{3}$ physically allowed temperature profiles. Because the errors in temperature are quite high we limit the range in which the temperature at a certain step in the profile, $T[i]$, is randomly set. To facilitate this, each temperature $T[i]$ is restricted to lie within a window with a size of $2 \mathrm{keV}$, the center of the window having the same relative distance to the upper and lower boundary of the spectroscopically derived temperature distribution as $T[i-1]$. Even though the observed temperature profile shows a strong gradient, the Monte-Carlo method produces physically correct temperature profiles (e.g., increasing mass with increasing radius) that cover almost the whole range of the temperature profiles allowed by the observations.

\begin{tabular}{c|c|c}
$\mathrm{r}$ & $\mathrm{T}(\mathrm{r})$ & $\mathrm{T}(\mathrm{r})$ \\
\hline$[\mathrm{kpc}]$ & {$[\mathrm{keV}]$} & {$[\mathrm{keV}]$} \\
\hline $0-445$ & $11.0-14.1$ & $10.5-16.2$ \\
$445-953$ & $8.6-14.2$ & $8.6-18.4$ \\
$953-2000$ & $2.9-4.9$ & $2.9-5.5$ \\
\hline
\end{tabular}

Table 1: The temperature profile for A2163 used as input for our mass determination. The right panel shows the temperature profile obtained by MMIYFT fitting ASCA and GINGA data simultaneously. Our conservative errors (see also text) are centered on MMIYFT's best fit data. The left profile is obtained by MMIYFT using only ASCA data. Using an updated version of the GIS effective area, the results based only on ASCA data yield higher temperatures, more in agreement with the combined fit (Markevitch, private communication). 
Our derived mass profile for A2163 is in general agreement with those derived by EAB and MMIYFT (which relied on the mass estimation method described by Hughes (1989), and Henry et al. (1993)). There are some slight differences between our results with the previous studies. For example, MMIYFT's mass estimates are a bit lower than our results when renormalized to $H_{0}=100$, with values of $1-1.5 \times 10^{15} M_{\odot}$ at a radius of roughly $1 h^{-1} \mathrm{Mpc}$ (although we note that the estimates formally agree within the experimental uncertainties). The difference probably arises due to the assumption of the shape of the total mass distribution combined with the observed steep temperature gradient. We compare our mass estimates with those derived by EAB. At the upper end of the region allowed by the statistical uncertainties, our mass estimate is in agreement with EAB's. However, we permit a lower mass value than EAB. We ascribe this difference as being due to the different temperature profile EAB employed (their temperature profile does not have such a steep temperature drop at the outer boundary of the cluster, so that their lower limit on the mass is higher).

We also calculated the mass distribution using the $\beta$ fit values of the HRI data in combination with MMIYFT's temperature profiles. In the outer regions the HRI results differ from the PSPC data. However, within an inner radius of $1 h^{-1} \mathrm{Mpc}$ the integrated and along the line-of-sight projected total mass (necessary for the comparison with the weak lensing) of the cluster varies only by a factor less than about $10 \%$ for the best fit value in $r_{c}$ and $\beta$. This shows again that the different values for $\beta$ and $r_{c}$ do not seriously affect the mass profile. The total gas masses derived by the HRI data agree within the error bars with the PSPC gas mass results as could be expected from the agreement found for the surface brightness profile parameters.

To facilitate a comparison between the weak lensing result and the X-ray results, we projected the radial mass profile derived from the Monte-Carlo technique onto the plane perpendicular to the plane of sight. The results are shown in Figure 8. In the two panels we show the results obtained by choosing $\beta, r_{c}$ values that lie at the extremes of the errors for the PSPC fit values. The errors shown are $\pm 2 \sigma$, calculated for the $10^{3}$ simulations. The effects of the uncertainties in the isothermal $\beta$-model are small, changing the radial mass profile by $9 \%$ at any radius, and the projected total mass by less than $4 \%$. The effect of the variation in the temperature profiles is larger, yielding $\mathrm{a} \simeq 20 \%$ increase in the total mass estimates, when the new temperature profile is used, although the results are consistent within the statistical uncertainties.

Finally, in Figure 9 we plot the gas-to-total mass fraction for A2163 using only the X-ray data. The gas mass determination based on ROSAT observations is quite insensitive to the temperature. To derive the gas mass profile, we employed the PSPC fit with the errors in $r_{c}$ and $\beta$ quoted above, and the corresponding best fit values for the central density. Comparing the total mass of the cluster with its gas mass yields a mean value of $M_{\text {gas }} / M_{\text {tot }} \simeq 0.07 h^{-3 / 2}$, with the statistical uncertainties allowing the range $0.02-0.16 h^{-3 / 2}$.

\subsection{Robustness of the $\mathrm{X}$-ray mass estimates}



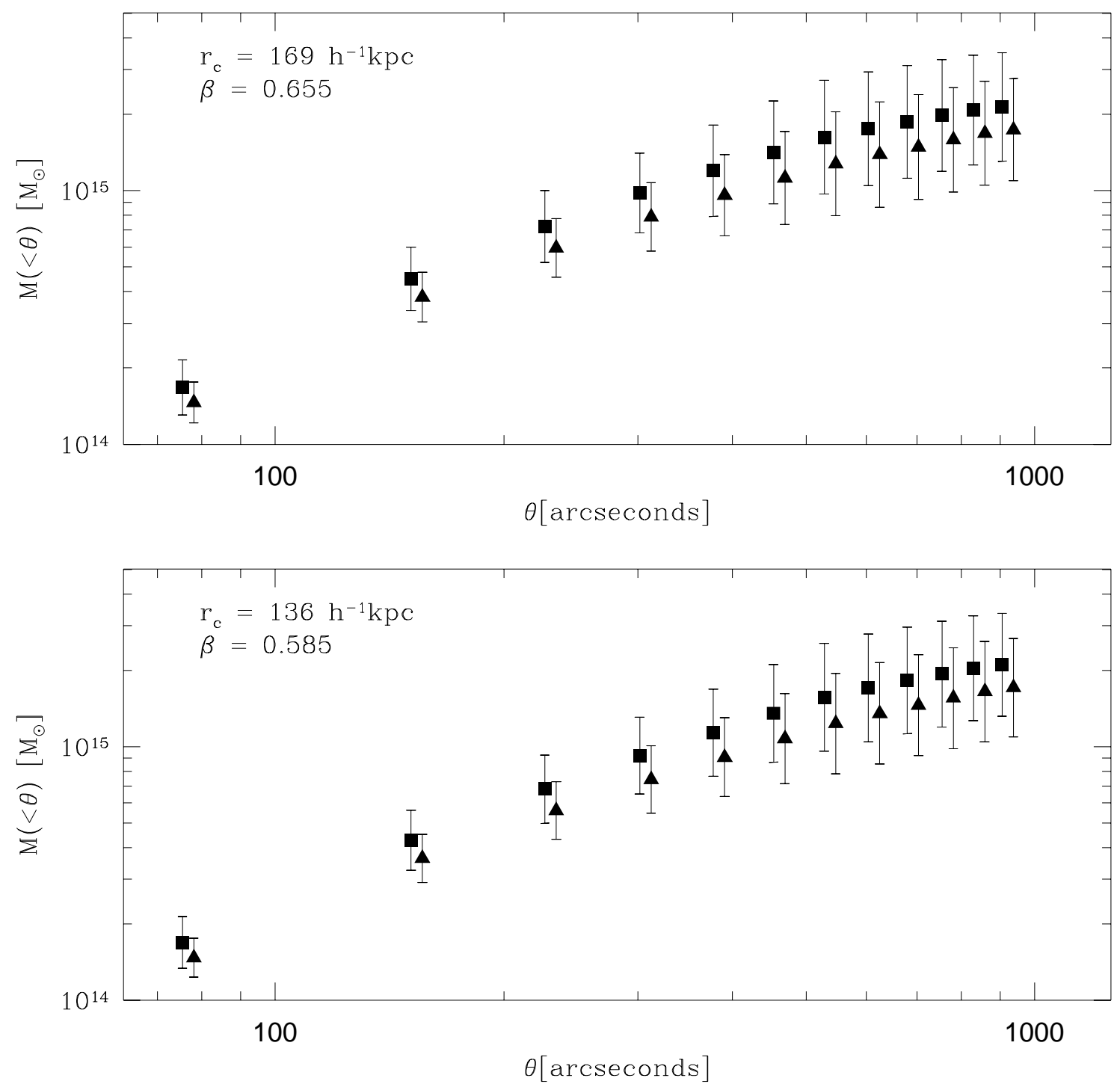

Fig. 8. - The projected mass profiles determined from the X-ray analysis. The temperature profiles are from MMIYFT: the squares are with the temperature parameters shown in column 2 of Table 1 while the triangles show the results allowing for a higher central temperature (see column 3, Table 1). The errors are $2 \sigma$ errors of the $10^{3}$ different randomly calculated temperature distributions. We integrate the mass profile out to a radius of $1920 h^{-1} \mathrm{kpc}$ over the line-of-sight. 


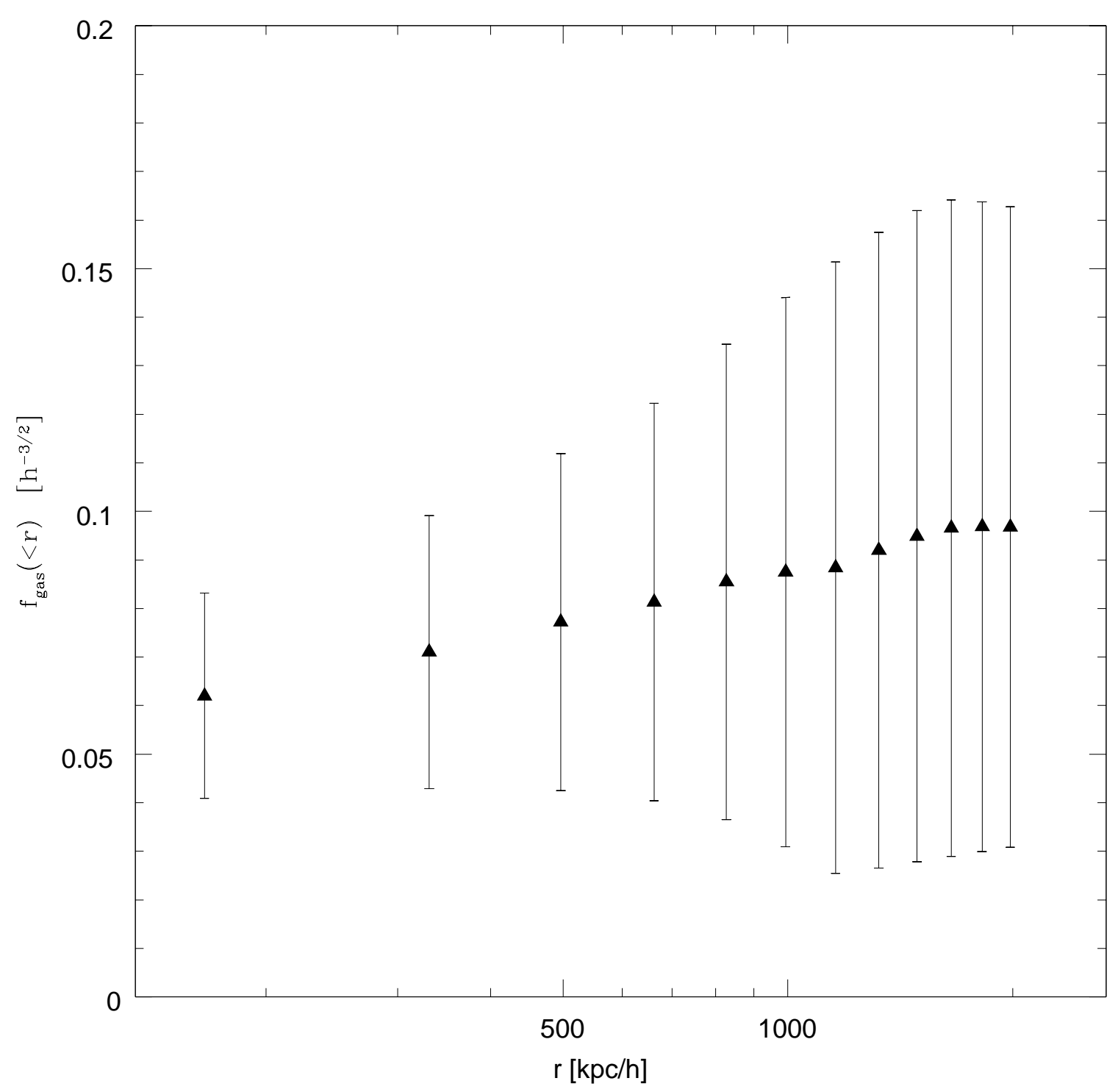

Fig. 9. - The projected gas mass fraction as a function of radius from the X-ray emission centroid. The total mass estimate used was determined by averaging over the mass estimates for the various $\beta$-fit parameters and two temperature profiles. For the central gas density, we employed the best fit values from the different fits. The errors were determined adding the uncertainties of the cumulative total and gas mass estimates in quadrature and hence the uncertainties of different points are correlated.The projected gas mass fraction as a function of radius from the X-ray emission centroid. The total mass estimate used is determined by averaging over the mass estimates for the various $\beta$-fit parameters and two temperature profiles. For the central gas density we take the best fit values for the different fits. 
Given all the evidence that A2163 is not a well relaxed system, the question may arise as to how this will effect the X-ray mass determination which depends on the assumption of hydrostatic equilibrium. This is in fact a quite general problem in the studies of clusters of galaxies: Since the formation time of clusters is comparable to the Hubble time, clusters are essentially still forming today. In particular, in a high density Universe, clusters are continuously accreting new material, quite often in a discontinuous fashion (e.g., Richstone et al. 1992; Navarro et al. 1995).

Various numerical simulation studies have been undertaken to determine whether the above assumptions introduce significant uncertainties in the mass estimates. In two recent simulation studies, Schindler (1996) and Evrard et al. (1996) analyzed the validity of the mass determination method used in X-ray analyses. In these studies the mass was determined using the same procedure we employed here, with the assumption that the gas is in hydrostatic equilibrium in the gravitational potential of the cluster. When the derived mass profiles were compared with the true mass profiles, the results were found to be, on the average, accurate within about 15 to $20 \%$, indicating that the mass determination from X-ray data is quite robust. As pointed out by Schindler (1996), larger deviations can occur particularly in those cases where a major merger shock wave passes through the cluster - the mass value can be well off by a factor of two locally, near the position of the shock wave - and in cases of major substructural features that are not properly accounted for.

There are some questions as to the accuracy of the simulations (Anninos \& Norman 1996). The important point that we wish to make here is that, at present, the uncertainties introduced by deviations from hydrostatic equilibrium and spherical symmetry are unlikely to be as large as the uncertainties in the temperature profiles. Indeed, we quantify this large uncertainty by employing the Monte Carlo method to determine the cluster mass. In this cluster, the uncertainty in the temperature introduces a factor of $\simeq 2$ error in the mass estimate both on large as well as small scales.

We consider below (see $\$ 5$.) the possibility that the cluster has undergone a recent merger and we are observing it in the first rebounce phase. In this case, the simulations of Schindler \& Müller (1993) provide a numerical model to guide the interpretation of our results. At the time of core passage of the two subcluster centers, the major thermalizing shock wave is started from the center of the cluster. The time since core passage can be estimated to be of the order of 0.5 Gyrs, based on the relative positions of the galaxy and mass concentrations. In this time the major shock wave has traveled about $1 \mathrm{Mpc}$. The X-ray mass profile is, however, derived for the whole cluster volume and since this profile is found to be very smooth (and the temperature profiles used here do not provide the resolution to resolve the temperature structure of the shock wave in any case, contrary to the resolution available in the simulations), the mass results we determine here will be little affected by the local deviation at the shock front.

\section{Comparison of the mass, light and gas distributions}

The combined analysis of the optical, lensing and X-ray data permits a direct comparison of the distributions of the galaxies, the hot gas, and the gravitational mass in A2163. These distributions 
are shown in Figures 1 , 3 and 7 , respectively. The projected distribution of the galaxies, the light and the total mass are quite similar. They all have extended, irregular morphologies exhibiting a very strong east-west elongation accentuated by two peaks. One of the maxima is located near the central giant elliptical galaxy about which arcs have been detected and the other is found between this galaxy and another bright elliptical. The similarity between the light and the mass maps suggests that light is tracing the mass in this cluster. This assertion can be further demonstrated by a comparison of the azimuthally-averaged radial optical surface brightness profile and the azimuthally-averaged radial surface mass density profile. Both have a similar radial dependence that is consistent with $l(\theta), \Sigma(\theta) \propto \theta^{-0.4}$, which is shallower than isothermal. In Figure 6, we show the mass-to-light ratio for the cluster and find that it is constant with radius within the given uncertainties with an average value of $M / L_{V}=(300 \pm 100) h M_{\odot} / L_{V \odot}$.

The lensing mass distribution was determined independently from the V- and I-band data. Several interesting features arise from the shear measurements and the inversion for the 2D mass profile. First, as seen in Figure 4 , the radial shear profile is rather flat and is much weaker than that induced by a singular isothermal profile characterized by a velocity dispersion comparable to that observed for the cluster galaxies, even if one takes into consideration the possibility that shear in the inner $1^{\prime}$ might be higher than observed. On the other hand, a mass distribution also characterized by $\sigma=1680 \mathrm{~km} / \mathrm{s}$ but with a core radius comparable to that observed in the X-ray produces less shear than observed, particularly in the inner $1^{\prime}$ (and such a profile would not be able to produce the thin large arcs observed in the vicinity of the giant elliptical galaxy. Even if the cluster surface mass is flat over the range $1^{\prime}<\theta<2^{\prime}$, both the observed shear profile and the thinness of arcs suggest that the cluster mass profile must steepen in the central regions). A singular model that provides a reasonable fit to the measured shear corresponds to $\sigma=740 \mathrm{~km} / \mathrm{s}$ although a singular isothermal model with $\sigma$ as high as $1000 \mathrm{~km} / \mathrm{s}$ is also consistent with the observations, particularly if one takes into account the possible suppression of shear in the inner regions of the cluster by contamination. Indeed, under the simple assumption of a spherical, isothermal mass distribution, a velocity dispersion of $\simeq 930 \mathrm{~km} / \mathrm{s}$ would be required to form the Einstein radius at the observed arc position.

The result of the flat, extended nature of the shear profile is that the significance of the features seen in the 2D mass reconstruction is not very high (especially in comparison with other clusters we have studied). We do see some evidence for a bi-modal structure, with each peak being resolved at the $\simeq 3 \sigma$ level. The small scale deviations between the $2 \mathrm{D}$ mass reconstructions seen in the I- and V-band data can be partially attributed to a combination of seeing and small number statistics. Moreover, we wish to stress that if the cluster density profile consists of a large, very extended, relatively flat structure, with small scale density substructures supposed on the flat plateau, then we would expect to observe a small amplitude for the shear (in the limit of a constant surface density sheet, there is no shear generated). Hence, if this plateau extends right across our field of view, we would detect a weak shear signal and explain the relatively small significance of the mass peaks in the reconstructions. This is an observationally testable hypothesis with larger angular scale optical observations.

In contrast to the projected galaxy, light and total matter distributions, the ROSAT/HRI X-ray surface brightness profile has a much more regular appearance, and is a centrally peaked distribution. The surface brightness distribution has one maximum located between the two maxima of the optical light and projected mass distributions. The X-ray isophotes are elongated in the east-west direction but 
the elongation is much less pronounced than that seen in the distribution of light and mass. To some extent, a smoother distribution of the X-ray emission is theoretically expected because if the gas is in hydrostatic equilibrium within the cluster's gravitational potential, its distribution traces the potential, and the gravitational potential always has a smoother structure than the mass distribution (e.g., Binney \& Tremaine 1987; Buote \& Canizares 1996).

Like the surface mass density and the optical surface brightness profiles, the radial X-ray surface brightness profile is very shallow in the center but falls off more steeply at larger radii. The X-ray surface brightness profile is weighted by an integration of the gas density squared in the line of sight. Accounting for this square dependence, one finds a radial dependence of the projected gas density at large radii of $\propto \theta^{-0.8}$. In Figure 9, we show the gas mass fraction as a function of radius and find a mean value of $M_{\text {gas }} / M_{\text {tot }} \simeq(0.07 \pm 0.03) h^{-3 / 2}$.

In order to compare the X-ray and lensing mass results we recalculated the lensing mass profiles by taking the X-ray centroid as the center. We also averaged the two different X-ray mass profiles (shown in Figure 8) corresponding to the two extreme data sets for $\beta, r_{c}$, and the two temperature profiles. The lensing and X-ray mass profiles are compared in Figure 10. The data sets only overlap out to a radius of $200^{\prime \prime} \simeq 500 h^{-1} \mathrm{kpc}$ to which the lensing analysis is limited. In the overlap region the lensing mass estimates are systematically lower by a factor of $\simeq 2$ than the X-ray results, although formally the results are consistent with each other given the substantial uncertainties.

As we discussed in $\$ 2.2$, one important consideration to bear in mind is that the lensing mass estimate may be biased downward if the cluster mass extends into the control annulus used to calculate the $\zeta$-statistic. For A2163, this is a likely scenario as our optical observations cover a relatively small field of view. The shallow surface mass density profiles and the optical surface brightness profiles both suggest that the cluster is quite extended. In addition, the cluster X-ray emission has been traced to $2.2 h^{-1} \mathrm{Mpc}$. The only accurate, model-independent way to avoid the bias is to observe and analyze the weak lensing distortions in the cluster out to larger radii. In the absence of necessary observations, we can attempt to estimate the magnitude of the bias affecting the lensing data based on the analytic spherically symmetric models fit to the observed radial shear profile (see Figure 4 ). We find that the actual projected cluster mass could be anywhere from a factor 1-2 higher, depending on the assumed model for the mass distribution. Alternatively, we can start with the null hypothesis that the lensing and X-ray mass estimates should agree, determine, based on the X-ray mass profile, the mean surface mass density in the control annulus and correct the lensing $\zeta$ estimate at every radius by this amount. The results of applying such a "correction" is displayed in Figure 10. As expected, the weak lensing mass estimates increase and the agreement between the lensing and X-ray results improves.

Having found consistency between the two data sets, we can consider more closely the cluster mass-to-light ratio and the cluster gas mass fraction. If on large scales, the dark matter and the baryons are not segregated, and the clusters of galaxies accrete gas (baryons) and dark matter in an indiscriminant way during their formation, and the efficiency of galaxy formation is the same in all environments, then clusters of galaxies can be thought of as representative elements of the Universe. The cluster mass-to-light ratio and the gas mass fraction, therefore, should equal their respective universal values. Comparing the mass-to-light ratio of $M / L_{V}=(300 \pm 100) h M_{\odot} / L_{V \odot}$ for A2163 with mean value for the local Universe (see Carlberg et al. 1995) implies a value of $\Omega \simeq 0.3 \pm 0.1$. Similarly, comparing 


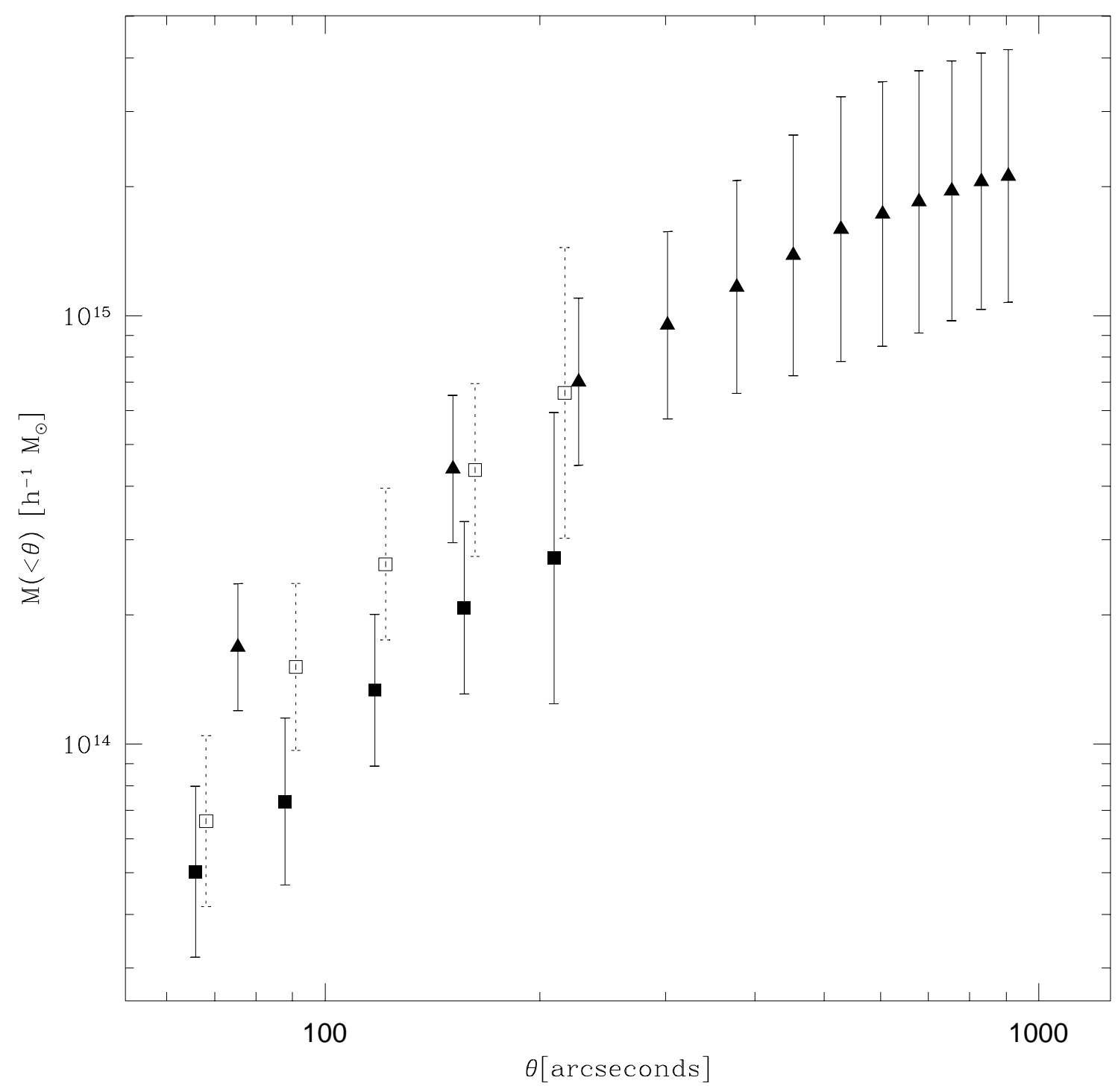

Fig. 10.- The radial mass profiles determined from the X-ray and lensing analysis. The triangles display the total mass profile determined from the X-ray data. The solid squares are the weak lensing estimates. The open squares are the lensing estimates "corrected" for the mean surface density in the control annulus determined from the X-ray data. The conversion from angular to physical units is $60^{\prime \prime}=0.127 h^{-1} \mathrm{Mpc}$. 
A2163's gas mass fraction of $M_{\text {gas }} / M_{\text {tot }} \simeq(0.07 \pm 0.03) h^{-3 / 2}$ with the universal mean baryon fraction of $f_{b} \leq 0.015\left(\Omega h^{2}\right)^{-1}$ predicted by nucleosynthesis (Walker et al. 1991) also implies $\Omega \leq 0.4 h^{-1 / 2}$. Both estimates point towards a low density Universe provided assumptions noted above are valid.

\section{Interpretation of the dynamical state of the cluster}

Taken jointly, the differences in the distributions of the cluster light, gas and dark matter strongly suggest that far from being a relaxed cluster, A2163 is very much a system in the process of formation. This conclusion was noted previously by EAB from a study of the overall galaxy distribution and the X-ray morphology of the PSPC image. For example, one striking feature in A2163 is that the X-ray emission centroid is so significantly devoid of any optical counterpart. The "cluster in formation" hypothesis is further supported by the cluster's very extended velocity histogram, a high velocity dispersion and the fact that coordinate-redshift galaxy distribution shows significant substructure (Soucail et al. 1996). The extended velocity histogram and the high velocity dispersion is most likely due to velocity distributions of infalling subclusters overlapping in velocity space. The value of $\beta_{T}=\sigma^{2} /\left(k T / \mu m_{p}\right)=1.3-1.5$ is larger than the canonical value of 1 and the values found in the range 1 to 1.2 for fairly quiescent clusters indicating that A2163 is not in a relaxed dynamical state. Furthermore, the presence of a luminous extended radio halo (Herbig \& Birkinshaw 1995) can be taken as yet another signature of the cluster having suffered a recent merger event. Very few clusters have large radio halos associated with them and all those that do (e.g., A2256, A2255 or Coma) show signatures of recent merger (Tribble 1993; Burns et al. 1994; Briel \& Henry 1994; Feretti \& Giovannini 1996). Theoretical arguments further strongly favor the extended radio halo/merger connection (see Böhringer et al. 1992; Böhringer 1995). Finally, evidence of complex, small-scale temperature variations in the central regions of the cluster described by Markevitch et al. (1994) as well as the extremely steep drop in the the mean gas temperature between the central regions and the outer regions (MMIYFT), provide further evidence that the cluster, as a whole, is unrelaxed. Other clusters with temperature substructure such as A754 (Henry \& Briel 1995) and A2256 (Briel et al. 1991; Briel \& Henry 1994; Roettiger, Burns \& Pinkney 1995) show evidence of recent/ongoing merger.

In the picture described above, the two major peaks in the light distribution probably correspond to the two most dominant subclusters that are in the process of merging (see also EAB). One scenario that describes the relative dark matter and gas distributions is that the two colliding subunits of the cluster have already passed through each other and are on their second turn-around trajectory. In this interpretation the two maxima of the galaxy distribution may be relics of the central regions of the two former subclusters. The hypothesis is substantiated by comparison with numerical simulations of cluster formation. In the N-body/hydrodynamical simulations by Schindler \& Müller (1993) for example the central regions of the two subclusters are traced by distinct maxima in the mass particles and the X-ray distribution during most of the first infall period. During the rebounce phase, after the central passage, the former subcluster centers are still recognizable as clumps of mass particles for some time while the X-ray surface brightness distribution appears smooth and not multi-peaked. At this state the simulated clusters resemble very much A2163 in appearance. Another well studied cluster which is most likely found in a similar state is A2255. In this case the X-ray peak is separated from the two dominant central 
galaxies and the cluster shows an elongation in the central region and an overall elliptical shape Burns et al. 1994; Feretti \& Giovannini 1996). From simulation of the cluster merger carried out to match and explain the morphology of A2255, Burns et al. (1994) concluded that the cluster is in a rebounce phase.

This comparison of the observational features of A2163 with results from simulations can of course not provide a unique identification of the dynamical state of the cluster. In this case they seem to provide a very plausible scenario, however, which gains further support for example by the presence of the radio halo. It will be quite difficult, on the other hand, to substantiate this picture purely by more observational data.

\section{Summary}

We have studied optical, gas and dark matter distributions in the cluster of galaxies Abell 2163. We have traced the cluster galaxy light distribution over a $7^{\prime} \simeq 1 h^{-1} \mathrm{Mpc}$ field centered on the dominant central galaxy. Using the weak gravitational distortion of background galaxies and correcting for systematic effects that can bias the galaxy shapes, we have mapped the dark matter distribution in the cluster over the same scale. Combining the observed X-ray surface brightness profile from ROSAT/PSPC observations with the spectroscopically determined temperature profiles, we have estimated the projected total mass and gas mass distribution to a radius of $\simeq 2 h^{-1} \mathrm{Mpc}$. In the present case, we find agreement at the $2 \sigma$ level between the raw weak lensing and X-ray inferred masses, with the $\mathrm{X}$-ray mass determinations being a factor of $\simeq 2$ higher. Correcting for matter in the control annulus, as determined from the X-ray observations, yields a much better agreement between the two results. The extended nature of the mass distribution is consistent relatively weak shear profile that was measured in this cluster. The extended and clumpy nature of the cluster galaxy light distribution, the broad cluster galaxy velocity histogram, the high value of $\beta_{T}$, the extended luminous radio halo, and the irregular mass distribution inferred from the weak lensing analysis, suggests that A2163 is a cluster in the process of formation.

The observations presented here have been a useful check of the consistency between the two mass determination methods and have enabled us to speculate on the dynamical state of the cluster. The somewhat unsatisfactory aspect of this is that, at least for the lensing analysis, the mass determinations were confined to a relatively small physical radius from the cluster center. Clearly, any conclusions about the universality of the gas mass fraction and mass-to-light ratio are subject to interpretations regarding the physics of clusters centers, and how representative a remarkable cluster like A2163 is of the Universe as a whole. A more pleasing comparison should, and now could, be done at over larger scales. Indeed, the technology now exists for such large field lensing observations with the MOCAM 14' and the $\mathrm{UH} \simeq 0.5^{\circ}$ cameras at CFHT. One exciting example is that, in hierarchical clustering scenarios, clusters of galaxies tend to form at intersections of filaments. In clusters such as A2163, which appear to be very much in formation, it may be possible to use large field observations of the weak lensing to detect filaments along which mass is flowing into the cluster. With the types of observations possible with the current generation of instruments, a wide range of outstanding issues can be probed regarding the dark matter content and distribution in the Universe. 
We would like to thank Sabine Schindler and Rien van de Weygaert for many enlightening discussions. It is a pleasure to acknowledge the valuable assistance of Richard Griffiths and the MDS team. We also gratefully acknowledge the redshift data and extrapolation to faint magnitudes provided by Simon Lilly, Caryl Gronwall, and David Koo. AB gratefully acknowledges support from The Dudley Observatory. 


\section{REFERENCES}

Abell, G.O., Corwin, H.G., \& Olowin, R.P. 1989, ApJS, 70, 1

Anninos, P., \& Norman, M. L. 1996, ApJ, 459, 12

Arnaud, M , Elbaz, D., Böhringer, H., Soucail, G., \& Mathez, G. 1994, in New Horizons of X-ray Astronomy, eds. F. Makino \& T. Ohashi (Tokyo: Universal Academy Press), 537

Arnaud, M., Hughes, J.P., Forman, W., Jones, C., Lachieze-Rey, M., Yamashita, K., \& Hatsukade, I. 1992, ApJ, 390, 345

Babul, A., \& Katz, N. 1993, ApJ, 406, 51

Bessell, M.S. \& Brett, J.M. 1988, PASP, 100, 1134

Binney, J., \& Tremaine, S. 1987, Galactic Dynamics, (Princeton: Princeton University Press)

Böhringer, H. 1995, in Reviews in Modern Astronomy 8: Cosmic Magnetic Fields, ed. G. Klare (Springer Verlag), 259

Böhringer, H. 1994, in Proceedings of the NATO Advanced Study Institute on Cosmological Aspects of X-ray clusters of Galaxies, ed. W. Seitter (Boston: Kluwer Academic Publishers), 123

Böhringer, H., Schwarz, R.A., Briel, U.G., Voges, W., Ebeling, H., Hartner, G., \& Cruddace, G., 1992, in Clusters and Superclusters of Galaxies, ed. A.C. Fabian, (Dordrecht: Kluwer Academic Publishers), 71

Blumenthal, G.R., Faber, S.M., Primack, J.R., \& Rees, M. 1984, Nature, 311, 517

Briel, U.G., \& Henry, J.P. 1994, Nature, 372, 439

Briel, U. G., Henry, J.P., Schwarz, R., Böhringer, H., \& Ebeling, H. 1991, A\&A, 246, L10-13

Buote, D., \& Canizares, C. 1996, ApJ, 457, 565

Burns, J.O., Roettiger, K., Ledlow, M. \& Klypin, A. 1994, ApJ, 427, L87

Carlberg, R., Yee, H.K.C., Ellingson, E., Abraham, R., Gravel, P., Morris, S., \& Pritchet, C. 1995, preprint

Coleman, G.D., Wu, C.C., \& Weedman, D.W. 1980, ApJ, 43, 393

David, L.P., Jones, C., \& Forman, W. 1995, ApJ, 445, 578

Davis, L. 1990, private communication

Efstathiou, G. 1995, in Les Houches Lectures on Galaxy Formation, Elsevier Science Publications, Netherlands

Elbaz, D., Arnaud, M., \& Böhringer, H. (EAB) 1995, A\&A, 293, 337

Evrard, A., Metzler, C., \& Navarro, J. 1995, preprint 
Fahlman, G., Kaiser, N., Squires, G., \& Woods, D. 1994, ApJ, 437, 56

Feretti, L., \& Giovannini, G. 1996, Proc of the IAU Symposium 175 on extragalactic radio sources, (in press)

Fort, B. \& Mellier, Y. 1994, A\&AR, 5, 239

Henry, J.P. \& Briel, U.G. 1995, ApJ, 443, L9

Henry, J.P., Briel, U.G., \& Nulsen, P.E.J. 1993, A\&A, 271, 413

Herbig, T., \& Birkinshaw, M. 1995, BAAS, 26, 1403

Holzapfel,W., Arnaud, M., Ade, P., Church, S., Fischer, M., Mauskopf, P., Rephaeli, Y., Wilbanks, T.M., Lange, A. 1996, ApJ, submitted

Hughes, J.P. 1989, ApJ, 337, 21

Jones, C., \& Forman, W. 1984, ApJ, 276, 38

Jones, C., \& Forman, W. 1992, Clusters \& Superclusters of Galaxies, ed. A.C. Fabian, (Dordrecht: Kluwer Academic Publishers), 49

Kaiser, N., \& Squires, G. 1993, ApJ, 404, 441

Kaiser, N., Squires, G., \& Broadhurst, T. 1995, ApJ, 449, 460

Kneib, J.P., Mellier, Y., Pello, R., Miralda-Escudé, J., Le Borgne, J.-F., Böhringer, H., Picat, J.-P. 1995, A\&A, 303, 27

Landolt, A. U. 1992, A\&A, 104, 340

Lilly, S.J. 1993, ApJ, 411, 501

Lilly, S.J. 1995, private communication.

Loewenstein, M. 1996, in proceedings of the 2nd international conference sponsored by UCLA on Sources and Detection of Dark Matter in the Universe, in press

Markevitch, M, Yamashita, K., Furuzawa, A., \& Tawara, Y. 1994, ApJ, 436, L71.

Markevitch, M, Mushotsky, R., Inoue, H., Yamashita, K., Furuzawa, A., \& Tawara, Y. (MMIYFT) 1995, ApJ, accepted

Miralda-Escudé, J. \& Babul, A. 1995, ApJ, 449, 18

Navarro, J., Frenk, C.S., \& White, S.D.M. 1995, MNRAS, 275, 720

Neumann, D.M., \& Böhringer, H. 1995, A\&A, 301, 86

Odewahn, S.C., Bryja, C., \& Humphreys, R.M. 1992, PASP, 104, 553

Richstone, D., Loeb, A., \& Turner, E.L. 1992, ApJ, 393, 477

Roettiger, K., Burns, J.O. \& Pinkney, J. 1995, ApJ, 453, 634 
Sarazin, C. 1986, Rev. Mod. Phys., 58, 1

Schindler, S. 1995, A\&A, 305, 756

Schindler, S., \& Müller, E. 1993, A\&A, 272, 137

Smail, I., Ellis, R.S., Fitchett, M.J., \& Edge, A.C. 1995, MNRAS, 273, 277

Soucail, G., Arnaud, M., \& Mathez, G. 1996, in preparation

Squires, G., \& Kaiser, N. 1995, submitted to ApJ

Squires, G., Kaiser, N., Babul, A., Fahlman, G., Woods, D., Neumann, D. M., \& Böhringer, H. 1996, ApJ, accepted

Stetson, P. B. \& Harris, W.E. 1988, A\&A, 96, 909

The, L.S., \& White, S.D.M. 1986, AJ, 92, 1248

Tresse, L., Hammer, F., le Fevre, O., \& Proust, D. 1993, A\&A, 277, 53

Tribble, P. 1993, MNRAS, 263, 31

Tyson, J., Valdes, F. \& Wenk, R. 1990, ApJ, 349, L19

Walker, T.P., Steigman, G., Schramm, D.N., Olive, K.A., \& Kang, H. 1991, ApJ, 378, 186

White, D. A., \& Fabian, A. C. 1995, MNRAS, 273, 72

White, S. D. M. 1992, in Clusters and Superclusters of Galaxies, ed. A.C. Fabian, (Kluwer: Dordrecht), 17

White, S.D.M., Navarro, J.F., Evrard, A.E., \& Frenk, C. S. 1993, Nature, 366, 429

Wilbanks, T.M., Ade, P.A.R., Fischer, M.L., Holzapfel, W.L., \& Lange, A.E. 1994, ApJ, 427, L72

This preprint was prepared with the AAS $\mathrm{LAT}_{\mathrm{E}} \mathrm{X}$ macros v3.0. 